\title{
MEEC: First Workshop on Momentary Emotion Elicitation and Capture
}

\begin{tabular}{|c|c|}
\hline $\begin{array}{l}\text { Abdallah El Ali } \\
\text { Centrum Wiskunde \& Informatica } \\
\text { Amsterdam, The Netherlands } \\
\text { aea@cwi.nl }\end{array}$ & $\begin{array}{l}\text { Mariam Hassib } \\
\text { Bundeswehr University Munich } \\
\text { Munich, Germany } \\
\text { mariam.hassib@ifi.Imu.de }\end{array}$ \\
\hline $\begin{array}{l}\text { Monica Perusquía-Hernández } \\
\text { NTT Communication } \\
\text { Science Laboratories } \\
\text { Atsugi, Japan } \\
\text { perusquia@ieee.org }\end{array}$ & $\begin{array}{l}\text { Alexander Meschtscherjakov } \\
\text { University of Salzburg } \\
\text { Salzburg, Austria } \\
\text { alexander.meschtscherjakov@sbg.ac.at }\end{array}$ \\
\hline $\begin{array}{l}\text { Pete Denman } \\
\text { Intel Corp } \\
\text { Portland, USA } \\
\text { pete.a.denman@intel.com }\end{array}$ & $\begin{array}{l}\text { Denzil Ferreira } \\
\text { University of Oulu } \\
\text { Oulu, Finland } \\
\text { denzil.ferreira@oulu.fi }\end{array}$ \\
\hline $\begin{array}{l}\text { Yomna Abdelrahman } \\
\text { Bundeswehr University Munich } \\
\text { Munich, Germany } \\
\text { yomna.abdelrahman@unibw.de }\end{array}$ & $\begin{array}{l}\text { Niels Henze } \\
\text { University of Regensburg } \\
\text { Regensburg, Germany } \\
\text { niels.henze@ur.de }\end{array}$ \\
\hline
\end{tabular}

Permission to make digital or

hard copies of part or all of this work for personal or classroom use is granted without fee provided that copies are not made or distributed for profit or commercial advantage and that copies bear this notice and the full citation on the first page. Copyrights for third-party components of this work must be honored. For all other uses, contact the owner/author(s). CHI '20 Extended Abstracts, April 25-30, 2020, Honolulu, HI, USA.

Copyright is held by the author/owner(s).

ACM ISBN $x x x-x-x x x x-x x x x-x / x x / x x$.

http://dx.doi.org/xx.xxxx/xxxxxxx.xxxxxxx

\begin{abstract}
Recognizing human emotions and responding appropriately has the potential to radically change the way we interact with technology. However, to train machines to sensibly detect and recognize human emotions, we need valid emotion ground truths. A fundamental challenge here is the momentary emotion elicitation and capture (MEEC) from individuals continuously and in real-time, without adversely affecting user experience. In this first edition of the one-day CHI 2020 workshop, we will (a) explore and define novel elicitation tasks (b) survey sensing and annotation techniques (c) create a taxonomy of when and where to apply an elicitation method.
\end{abstract}

\section{Author Keywords}

emotion; affective computing; elicitation; capture; momentary

\section{CCS Concepts}

-Human-centered computing $\rightarrow$ Human computer interaction $(\mathrm{HCl}) ; \mathrm{HCl}$ design and evaluation methods;

\section{Background}

In the April 2018 issue of the Communications of the ACM, it was highlighted that "the ability to recognize human emotions and then respond appropriately - is essential to the true success of digital assistants and the many other artificial intelligences (Als) we interact with every day" [20]. However emotions are often private to an individual, and do not always 
have a direct overt manifestation (e.g., in facial expressions [4]). From a social, interactionist view, emotions can be viewed as dynamic, culturally mediated, and socially constructed experiences [5]. Indeed, as Barrett [3] states, in the absence of an objective, external way to measure emotional experience, we can only examine emotions through self-reports, and it is our role as researchers to ensure that our ratings are useful and valid indicators of what a person is experiencing. To this end, recognizing human emotions necessitates the ability to reliably and ethically elicit emotional (affective) states, and capture them computationally in order to serve as ground truths for the detection and recognition systems we develop [6].

There has been a rich history of emotion elicitation (or induction) methods [16], ranging from imagination, film, sound, music, images, to dyadic interactions, and even Virtual Reality (VR) environments. Indeed, while VR environments show great promise of increased immersion and sense of presence in example eliciting fear responses [23], users' sense of presence does not necessarily always result in a significant difference during self-reports in or outside VR [28]. Other experimental methods to elicit natural emotional responses include manipulations to elicit spontaneous versus posed smiles [10], such as asking users to watch funny videos and asking them to withhold from laughing, thus naturally eliciting genuine smiles. Such subtle microexpressions can be measured through facial distal electromyography (EMG) [25].

With respect to capture, previous work has shown the potentia of improving smartphone interactions using emotional facial expressions [15] and heart-rate data [18]), detecting engagement at museums using Brain-Computer Interfaces [1]), automatically detecting complex emotion constructs such as sympathy from foreign news media text [14], to automatically detecting learner affect in classrooms using thermal imaging [2]. Others have investigated what are called Experience Sampling
Methods which collect context data (location, temperature, etc.) to intelligently nudge users to fill in experience reports (e.g., AWARE framework [17]), with further investigations on the response and recall accuracy rates of varying the scheduling of self-reports [29]. Furthermore, there has been shown differences in ESMs across devices [19], which result in differences in response times across devices, as well as tradeoffs in interaction types, screen size, and device familiarity that can affect both users' experience and the reports made by users.

The foregoing notwithstanding, we face a fundamental challenge concerning temporal resolution in emotion elicitation and measurement: even though emotions, whether microexpressions or bodily changes, are in continuous flux and can be measured, self-reports do not have the same temporal resolution. Several factors contribute to this temporal resolution mismatch: different awareness (interoception) levels across individuals [8]; non-linearity in time perception [30]; and how emotions themselves alter time perception [12]. In this workshop, we address this challenge of Momentary Emotion Elicitation and Capture (MEEC) from individuals continuously and in real-time, without adversely affecting user experience. Specifically, we seek to more closely examine and define this wave of momentary elicitation and capture.

\section{MEEC Challenges}

While other workshops ${ }^{1}$ and dedicated conferences (cf., Affective Computing \& Intelligent Interaction) focus on the many technical challenges of implementing emotion recognition (e.g., using deep learning methods), less attention has been devoted into eliciting emotions as they occur, and how to capture them continuously and in real-time to serve as valid ground truths for

\footnotetext{
1Examples:
Design for Affective Intelligence @ ACII2017

Affective Computing in $\mathrm{HCl} @ \mathrm{HCl} 2018$

Emotion Recognition in the Wild Challenge (EmotiW) @ ICMI2019)
} 
the analyses proposed in such venues. Challenges are split across elicitation and capture, and include:

Elicitation: Which multi-modal (e.g., film, music) and multisensory (e.g., auditory, taste, olfactory) elicitation methods are most suitable for which contexts? What are the peculiarities across domains (e.g., understanding mobile interaction with and within automated vehicles [22]? How can we leverage the immersiveness of VR technologies for use as an elicitation method (cf., [24]), and what limits does this impose on capture? How do we elicit emotional states over time (e.g., mood)? What ethical considerations in elicitation [6] need to be considered to ensure we respect the users' personal, cognitive, and emotional boundaries?

Capture: How can we capture a wider range of human emotions / feelings / moods, in the moment that they occur? While methods are being developed to collect in situ affect data [9], challenges remain in the range of emotions and moods we can capture [7]. Importantly, which emotions should we capture [11] and how do cross-cultural differences impact this [21]? What emotional models do we draw upon, discrete e.g., Ekman's six basic emotions [13] or dimensional, e.g., Russell's Circumplex model [26]? Which annotation modalities (e.g., speech, gestures) and tools (e.g., questionnaires, ESMs) are most apt? Which devices (e.g., mobile, wearable) and sensors (e.g., RGB / thermal cameras, EEG) provide a good trade-off between unobtrusiveness and accurate measurements? How can we factor in attentional considerations (e.g., interruptions) to lower dropoff rates and improve self-reports in ESMs?

As a community of $\mathrm{HCl}$ researchers, we need to steer research addressing the slippery problem of elicitation and capture of emotions in the moment. Together with the Affective Computing community, we need to concretely define the collection and capture of valid emotion ground truths as an agenda and goal for the $\mathrm{CHI}$ community. Thus, the overarching goal of the first edition of the MEEC workshop is to establish lasting and meaningful connections across research communities concerned with affective computing, and to bring together students and researchers from various disciplines who are working on studying, designing, building, and/or evaluating the capture, prediction, and communication of human emotions.

\section{Pre-workshop Plans}

Participants and Workshop Publicity

We aim for the workshop to be interdisciplinary by nature, addressing academic researchers as well as industry. We expect participants from the areas of $\mathrm{CHI}$, ACI, UbiComp, ICMI, but also related areas including (cognitive) psychology and industrial design. The program committee comprises researchers and practitioners active in these research areas and who, moreover, plan to encourage participants from their institutes to submit to this workshop. This would ensure active participation in the preparation and execution of the workshop. We plan to encourage young scientists and $\mathrm{PhD}$ students to explore their research topics with domain experts. The call for papers will be distributed through the ACM SIGCHI mailing list, and further promoted though a website we will set up, as well as social networks (e.g., Twitter, Facebook). We expect approximately 15-20 participants, where 20 is the maximum. Furthermore, we will request participants to read all accepted submissions prior to the workshop day.

The workshop results will be made available on the workshop website (https://meec-ws.com) as well as in dedicated proceedings. This will help attract higher quality submissions, and allow for increased exposure and impact of the workshop before and after the event.

Key dates

- Call out: 11 December 2019

- Submission deadline: 11 February 2020 
- Notification of acceptance: 28 February 2020

- Workshop day: Saturday 25 or Sunday 26 April 2020

\section{Workshop Plan}

We plan for a full one-day workshop with submissions as position papers (2-8 pages), demos, and/or posters (A0 format) focusing on novel concepts, perspectives, or works in progress.

An important goal is to attract high-quality submissions spanning multiple research disciplines to encourage and shape the discussion on momentary emotion elicitation and capture. Also important is that the workshop will be highly interactive and group based in order to create a sense of community. Proposed schedule is shown in Table 1.

For the elicitation part, we will start the day with lightening talks to set the stage and inspire participants. By including a poster and demo session alongside lightening talks, we aim to ensure focused and lively discussion. These topics will be used as inspiration for the interactive elicitation method sketching part.

Figure 1: Printouts of Russell's Circumplex model of emotions [26] (adapted) will be used as a probe.
This will be in 4-5 groups, where each group will sketch out a series of novel momentary elicitation techniques by each focusing on a single human sensation (e.g., visual, olfactory) and 2-3 emotions to be evoked (see Figure 1). These can span multiple modalities and domains. Afterwards, each group will share be asked to demonstrate their elicitation technique(s) on one of us. This will involve role-playing, pantomime, storytelling, sensory stimulation, digital media (in AR/VR), or any means by which our emotions can be elicited (so long as they respect ethical boundaries within and beyond the WS).

For the capture part of the workshop, as organizers we will start by providing a survey of current sensing and annotation techniques. We will open the floor for discussion so as to allow participants to fill in knowledge gaps. With this survey of work fresh in our minds, we will begin the mapping session. In groups, each group will be asked to consider which elicitation method applies when, where, and with which sensing / annotation technique it is most suitably paired. Groups will be divided by modalities, and will be asked to chart out this using colored sticky notes. These mappings will be transferred to digital form and later shared on the WS website. To facilitate the workshop activities we would require the following resources: A0 poster pin-up boards and pins or glue tack, flipchart boards for 3-4 groups, flexible table arrangements, different colored post-its, pens, and color markers.

Submissions will be reviewed by the workshop organizers. Participants will be selected based on the Extended Abstract reviews for quality, novelty and inspirational aspects, aiming for a good balance of different perspectives on the topic of momentary emotion elicitation and capture. After the workshop, we will provide a summary report to be published on the website, an ACM Interactions contribution, and put the proceedings (in .pdf) online.

\section{Organizers and Program Committee}

Below are short biographies of each organizer. Expertise and interests are complementary and reflect the interdisciplinary perspectives of the workshop topic.

Abdallah El Ali (main contact) is a tenure-track researcher at the Distributed \& Interactive Systems group at Centrum Wiskunde \& Informatica (CWI) in the Netherlands. His research focuses on capturing, predicting, and communicating momentary human emotion and behavior. He combines advances in $\mathrm{HCl}$ and machine learning to enable pervasive affect sensing in and beyond mobile interactions. Website: https://abdoelali.com/

Monica Perusquía-Hernández is a Research Associate at NTT Communication Science Laboratories, Japan. Her research interests include affective computing, bio-signal pro- 
Table 1: Proposed schedule

\begin{tabular}{|c|c|c|}
\hline & Time & Activity \\
\hline \multirow{7}{*}{ 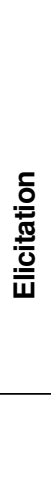 } & 9:00-9:10 & Brief welcome to the workshop \\
\hline & 9:10- 9:30 & $\begin{array}{l}\text { Speed dating session for participants, highlight- } \\
\text { ing (a) why they attended this workshop and (b) } \\
\text { sharing a story of own elicitation challenges }\end{array}$ \\
\hline & $9: 30-10: 30$ & Lightening talks (3-4 min. per participant) \\
\hline & $10: 30-11: 00$ & Coffee \& snacks break \\
\hline & $11: 00-12: 00$ & Elicitation method sketching (in 4-5 groups) \\
\hline & $12: 00-12: 30$ & Group presentations ( 7 min. per group) \\
\hline & $12: 30-14: 00$ & $\begin{array}{l}\text { Lunch (organizers will arrange joint lunch for } \\
\text { participants) }\end{array}$ \\
\hline \multirow{6}{*}{ 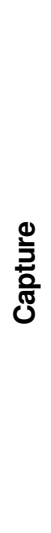 } & 14:00-14:30 & $\begin{array}{l}\text { Organizers give survey of sensing and annota- } \\
\text { tion techniques }\end{array}$ \\
\hline & $14: 30-15: 00$ & Open discussion about additional techniques \\
\hline & $15: 00-15: 30$ & Coffee \& snacks break \\
\hline & 15:30-16:30 & $\begin{array}{l}\text { Mapping session (in } 4-5 \text { groups): which elicita- } \\
\text { tion method applies when, where, and paired } \\
\text { with which sensing / annotation technique }\end{array}$ \\
\hline & $16: 30-17: 00$ & $\begin{array}{l}\text { Wrap up on workshop output, actions for follow- } \\
\text { up activities }\end{array}$ \\
\hline & $17: 00+$ & $\begin{array}{l}\text { After workshop wrap up and exchange of con- } \\
\text { tacts, further informal discussion continues } \\
\text { during drinks and dinner }\end{array}$ \\
\hline
\end{tabular}

cessing, augmented human technology, and artificial intelligence. In particular, she is interested in sensing techniques such as Computer Vision, EMG and Skin Conductance for congruence estimation between facial expressions and emotions when assessing user experience.
Pete Denman is a Senior Designer developing exploratory prototypes in "Labs" at Intel Corp. Focus on capturing emotion in daily life. He guides ideas through the design process, working with developers, engineers, and ethnographic researchers to create future technologies. Known best for being the lead designer at Intel for famed physicist Stephen Hawking's speech software, Denman could relate to Hawking's circumstances more than most due to his disability. Pete has a passion for connecting the areas of disabilities and learning/education with technology. Denman's current work focuses on affective computing and robots.

Yomna Abdelrahman is a postdoctoral researcher at the Bundeswehr University Munich in Germany. Her research focuses on Thermal Imaging operating in the far-infrared spectrum, novel interactive systems, engagement sensing and adaptive bio-sensor assistive systems. Recently, her research focus shifted to affective computing using thermal cameras as unobtrusive sensor.

Mariam Hassib is a postdoctoral researcher at the Bundeswehr University Munich in Germany. Her research interests are Brain Computer Interfaces (BCl), and Physiological Computing. She is working on building systems which provide feedback and adapt to the cognitive and affective states of users. She leverages the current ubiquity of $\mathrm{BCl}$ devices and wearable physiological sensors to create new tools that support cognitive-awareness outside the lab environment.

Alexander Meschtscherjakov is Associate Professor at the Center for Human-Computer Interaction of Salzburg University. $\mathrm{He}$ is focusing on persuasive interaction technologies, automotive user interfaces, contextual user experience and challenges of automation for $\mathrm{HCl}$ with a special focus on affective computing. He has co-organised conferences such as Persuasive '15 and more than 25 workshops (e.g., CHI 2019 [27]). 
Denzil Ferreira is an Associate Professor in Mobile Computing. His research interests are on technology-driven human behavior sensing and modeling, juxtaposing methods from large-scale data analysis, sensor instrumentation, applied machine learning, mobile and ubiquitous computing to understand and study a variety of human behavioral and social phenomena in naturalistic settings.

Niels Henze is professor for Media Informatics at the University of Regensburg. His research interests are mobile humancomputer interaction and pervasive computing. Particularly, he is interested in large-scale studies using mobile application stores as a research tool, interlinking physical objects and digital information, and multimodal interfaces. His recent work focuses on identifying, manipulating and evaluating how our sense of presence is affected in virtual reality environments.

\section{Call for Participation}

To train machines to sensibly detect and recognize human emotions, we need valid emotion ground truths. A fundamental challenge here is the momentary emotion elicitation and capture (MEEC) from individuals continuously and in real-time, without adversely affecting user experience. In this one-day $\mathrm{CHI} 2020$ workshop, we will (a) explore and define novel elicitation tasks (b) survey sensing and annotation techniques (c) create a taxonomy of when and where to apply an elicitation method.

We seek contributions across disciplines that explore how emotions can be naturally elicited and captured in the moment. Topics include:

\section{Elicitation:}

- multi-modal (e.g., film, music) and multi-sensory (e.g., auditory, taste, olfactory) elicitation

- emotion elicitation across domains (e.g., automotive, healthcare)
- elicitation and immersiveness (e.g., AR, VR)

- elicitation over time (e.g., mood)

- ethical considerations

\section{Capture:}

- emotion models (dimensional, discrete)

- annotation modalities (e.g., speech, gestures) and tools (e.g., questionnaires, ESMs)

- devices (e.g., mobile, wearable) and sensors (e.g., RGB

thermal cameras, EEG)

- attention considerations (e.g., interruptions)

\section{Submission}

We invite position papers, posters, and demos (2-8 pages, incl. references) that describe/showcase emotion elicitation and/or capture methods. Submissions will be peer-reviewed by 2 peers, and selected on their potential to spark discussion. Submissions should be prepared according to the SIGCHI Extended Abstracts format and submitted in PDF through Easychair (https://easychair.org/conferences/?conf=meec2020). Accepted submissions will be made available on the workshop website. At least one author must register for the workshop and one day of the conference.

- Deadline: 11 February 2020

- Notification: 28 February 2020

- Workshop: Saturday/Sunday, 25-26 April 2020

Website: https://meec-ws.com/

\section{REFERENCES}

[1] Yomna Abdelrahman, Mariam Hassib, Maria Guinea Marquez, Markus Funk, and Albrecht Schmidt. 2015.

Implicit Engagement Detection for Interactive Museums Using Brain-Computer Interfaces. In Proc. MobileHCl '15. ACM, New York, NY, USA, 838-845. DOI : http://dx.doi.org/10.1145/2786567.2793709 
[2] Yomna Abdelrahman, Eduardo Velloso, Tilman Dingler, Albrecht Schmidt, and Frank Vetere. 2017. Cognitive heat: exploring the usage of thermal imaging to unobtrusively estimate cognitive load. Proc. IMWUT '17 1, 3 (2017), 33.

[3] Lisa Feldman Barrett. 2004. Feelings or words. Understanding the content in self-report ratings of emotional experience. In Are emotions natural kinds? Perspectives on Psychological.

[4] Lisa Feldman Barrett, Ralph Adolphs, Stacy Marsella, Aleix M. Martinez, and Seth D. Pollak. 2019. Emotional Expressions Reconsidered: Challenges to Inferring Emotion From Human Facial Movements. Psychological Science in the Public Interest 20, 1 (2019), 1-68.

[5] Kirsten Boehner, Rogério DePaula, Paul Dourish, and Phoebe Sengers. 2007. How emotion is made and measured. IJHCS 65, 4 (2007), 275 - 291.

[6] R.A. Calvo, S. D'Mello, J. Gratch, and A. Kappas. The Oxford Handbook of Affective Computing.

[7] R. Cowie, E. Douglas-Cowie, N. Tsapatsoulis, G. Votsis, S. Kollias, W. Fellenz, and J. G. Taylor. 2001. Emotion recognition in human-computer interaction. IEEE Signal Processing Magazine 18, 1 (Jan 2001), 32-80. DOI : http://dx.doi.org/10.1109/79.911197

[8] Hugo D Critchley and Sarah N Garfinkel. 2017. Interoception and emotion. Current Opinion in Psychology 17 (oct 2017), 7-14.

[9] Pete Denman, Erica Lewis, Sai Prasad, Jennifer Healey, Haroon Syed, and Lama Nachman. 2018. Affsens: A Mobile Platform for Capturing Affect in Context. In Proc. Adjunct MobileHCl '18. ACM, New York, NY, USA, 321-326. DOI :

http://dx.doi.org/10.1145/3236112.3236158
[10] Hamdi Dibeklioğlu, Albert Ali Salah, and Theo Gevers. 2012. Are You Really Smiling at Me? Spontaneous versus Posed Enjoyment Smiles. In Computer Vision ECCV 2012, Andrew Fitzgibbon, Svetlana Lazebnik, Pietro Perona, Yoichi Sato, and Cordelia Schmid (Eds.). Springer Berlin Heidelberg, Berlin, Heidelberg, 525-538.

[11] Sidney D'Mello and Rafael A. Calvo. 2013. Beyond the Basic Emotions: What Should Affective Computing Compute?. In Proc. CHI EA '13. ACM, New York, NY, USA, 2287-2294.

[12] Sylvie Droit-Volet and Warren H. Meck. 2007. How emotions colour our perception of time. Trends in Cognitive Sciences 11, 12 (dec 2007), 504-513.

[13] Paul Ekman. 1992. An argument for basic emotions. Cognition and Emotion (1992), 169-200.

[14] Abdallah El Ali, Tim C. Stratmann, Souneil Park, Johannes Schöning, Wilko Heuten, and Susanne C.J. Boll. 2018. Measuring, Understanding, and Classifying News Media Sympathy on Twitter After Crisis Events. In Proc. CHI '18. Article 556, 13 pages.

[15] Abdallah El Ali, Torben Wallbaum, Merlin Wasmann, Wilko Heuten, and Susanne CJ Boll. 2017. Face2Emoji: Using Facial Emotional Expressions to Filter Emojis. In Proc. EA CHI '17. ACM, New York, NY, 1577-1584.

[16] Seyedeh Maryam Fakhrhosseini and Myounghoon Jeon. 2017. Chapter 10 - Affect/Emotion Induction Methods. In Emotions and Affect in Human Factors and Human-Computer Interaction, Myounghoon Jeon (Ed.). Academic Press, San Diego, 235 - 253.

[17] Denzil Ferreira, Vassilis Kostakos, and Anind K. Dey. 2015. AWARE: Mobile Context Instrumentation Framework. Frontiers in ICT 2 (2015), 6. DOI: http://dx.doi.org/10.3389/fict.2015.00006 
[18] Mariam Hassib, Daniel Buschek, Paweł W. Wozniak, and Florian Alt. 2017. HeartChat: Heart Rate Augmented Mobile Chat to Support Empathy and Awareness. In Proc. CHI '17. ACM, New York, NY, USA, 2239-2251. DOI : http://dx.doi.org/10.1145/3025453.3025758

[19] Javier Hernandez, Daniel McDuff, Christian Infante, Pattie Maes, Karen Quigley, and Rosalind Picard. 2016. Wearable ESM: Differences in the Experience Sampling Method Across Wearable Devices. In Proc. MobileHCl '16. 195-205.

[20] Marina Krakovsky. 2018. Artificial (Emotional) Intelligence. Commun. ACM 61, 4 (March 2018), 18-19.

[21] Daniel McDuff, Jeffrey M. Girard, and Rana el Kaliouby 2017. Large-Scale Observational Evidence of Cross-Cultural Differences in Facial Behavior. Journal of Nonverbal Behavior 41, 1 (01 Mar 2017), 1-19.

[22] Alexander Meschtscherjakov, Manfred Tscheligi, Peter Fröhlich, Rod McCall, Andreas Riener, and Philippe Palanque. 2017. Mobile Interaction with and in Autonomous Vehicles. In Proc. MobileHCl '17. ACM, New York, NY, USA, Article 78, 6 pages. DOI : http://dx.doi.org/10.1145/3098279.3119837

[23] Federica Pallavicini, Ambra Ferrari, Alessandro Pepe, Giacomo Garcea, Andrea Zanacchi, and Fabrizia Mantovani. 2018. Effectiveness of Virtual Reality Survival Horror Games for the Emotional Elicitation: Preliminary Insights Using Resident Evil 7: Biohazard. In Univ. Access in $\mathrm{HCl}$. Virtual, Augmented, and Intelligent Environments, Margherita Antona and Constantine Stephanidis (Eds.). Springer, 87-101.

[24] Xiaolan Peng, Jin Huang, Linghan Li, Chen Gao, Hui Chen, Feng Tian, and Hongan Wang. 2019. Beyond Horror and Fear: Exploring Player Experience Invoked by Emotional Challenge in VR Games. In Proc. CHI EA '19.
[25] Monica Perusquía-Hernández, Saho Ayabe-Kanamura, Kenji Suzuki, and Shiro Kumano. 2019. The Invisible Potential of Facial Electromyography: A Comparison of EMG and Computer Vision when Distinguishing Posed from Spontaneous Smiles. In Proc. CHI '19. ACM, New York, NY, USA, Article 149, 9 pages. DOI:

http://dx.doi.org/10.1145/3290605.3300379

[26] James A Russell. 1980. A circumplex model of affect. Journal of personality and social psychology 39, 6 (1980), 1161.

[27] Shadan Sadeghian Borojeni, Alexander Meschtscherjakov, Bastian Pfleging, Wendy Ju, Frank Flemisch, Christian P. Janssen, Andrew L. Kun, and Andreas Riener. 2019. Looking into the Future: Weaving the Threads of Vehicle Automation. In Proc. CHI EA '19. Article W08, 8 pages.

[28] Valentin Schwind, Pascal Knierim, Nico Haas, and Niels Henze. 2019. Using Presence Questionnaires in Virtual Reality. In Proc. CHI '19. ACM, New York, NY, USA, Article 360,12 pages. DOI :

http://dx.doi.org/10.1145/3290605.3300590

[29] Niels van Berkel, Jorge Goncalves, Lauri Lovén, Denzil Ferreira, Simo Hosio, and Vassilis Kostakos. 2019. Effect of experience sampling schedules on response rate and recall accuracy of objective self-reports. IJHCS 125 (2019), $118-128$.

[30] Gal Zauberman, B. Kyu Kim, Selin A. Malkoc, and James R. Bettman. 2009. Discounting time and time discounting: Subjective time perception and intertemporal preferences. Journal of Marketing Research 46, 4 (aug 2009), 543-556. 